\title{
LA INGENIERÍA FORENSE COMO HERRAMIENTA EN LA INVESTIGACIÓN DEL TERREMOTO DEL 16 DE ABRIL DEL 2016 OCURRIDO EN EL ECUADOR
}

\author{
T. MOROCHO \\ T. M. LL. Construcciones, Área de Patología e Ingeniería Forense. Quito, Ecuador.
}

\begin{abstract}
RESUMEN
El día 16 de abril de 2016, a las 18H58, se produjo en el Ecuador un sismo de 7,8 grados en la escala de Richter, cuyo epicentro se localizó entre las provincias de Esmeraldas y Manabí, generando importantes pérdidas humanas, así como daños severos en la infraestructura pública y privada de las provincias de Esmeraldas, Manabí, Santa Elena, Santo Domingo de los Tsáchilas. El objetivo de esta investigación es saber las causas que originaron fallas estructurales en las edificaciones. Mediante el uso de la metodología de Ingeniería Forense, lograremos determinar el comportamiento de un sistema dinámico y las consecuencias del daño originadas por tal suceso, las cuales son cuantificables a medida del grado de daño adquirido
\end{abstract}

Palabras Claves: Jaramijó, Rehabilitación, Infraestructura, Reparación.

\begin{abstract}
On April 16, 2016, at 18H58, an earthquake measuring 7.8 on the Richter scale occurred in Ecuador, whose epicenter was located between the provinces of Esmeraldas and Manabí, causing significant human losses, as well as damages Severe in the public and private infrastructure of the provinces of Esmeraldas, Manabí, Santa Elena, Santo Domingo de los Tsáchilas. An investigation was made of the origin and causes of the incident in the buildings, determining the circumstances for an undesired event to occur. By using the methodology of Forensic Engineering, we were able to determine the behavior of a dynamic system and the consequences of the damage originated by such event, which are quantifiable to measure the degree of damage acquired.
\end{abstract}

Keywords: Richter, Forensic Engineering, Ecuador, Damage 


\section{INTRODUCCIÓN}

Uno de los primeros casos documentados dentro de la Ingeniería Forense, es el puente DEE en Chester, Inglaterra, el cual se hundió mientras un tren lo cruzaba. El ingeniero que diseño el puente fue acusado de negligencia tras la investigación del desastre. Nadie construye una obra esperando que se venga al suelo y mucho menos que causen daño a alguien, pero los accidentes pasan y se esperan que alguien asuma la responsabilidad. Determinar quién es responsable ante una falla estructural es tarea que se le ha encargado al Ingeniero Forense. El Ingeniero Forense no es ni juez ni verdugo, su papel no es encontrar culpables si no encontrar causas y entregar soluciones. El reporte comprende el total de la investigación y se presenta a través de textos y gráficos. Comprende los antecedentes del proyecto, el incidente de la falla, las consideraciones del diseño, el análisis del modo y causas de la falla, incluyendo las hipótesis de las mismas. Podría incluir recomendaciones de subsanación.

\subsection{Ingeniería Forense}

\subsection{1 ¿Propósito?}

- Contribuir al proceso constructivo

- Identificar definiciones y/o defectos.

- Determinar le relevancia de los mismos.

- Recomendar soluciones eficientes al proceso constructivo.

\section{Definición de Ingeniería Forense}

Etimología latina: Forensis

$>$ Público.

$>$ Relacionado con los tribunales.

> Capacitado para la argumentación legal o pública.

Es una disciplina que aplica herramientas de varias ciencias e ingeniería para dos fines:

$>$ Preventivo: reduce el riesgo de fallo en un producto ingenieril y mejorar su rendimiento y su coste.

> Paliativo: reconstruir la secuencia de sucesos que conducen a una pérdida económica o a lesiones motivadas por fallo en un producto ingenieril, ayudando a determinar remedios y responsabilidades.

La definición más general sería: incapacidad de un componente, estructura o instalación para cumplir con su supuesto cometido.

\section{DESARROLLO}

El conocimiento actual sobre el efecto del sismo en las construcciones proviene de la observación de los daños, de los estudios de laboratorio y de rigurosos estudios analíticos. Estos conocimientos han impulsados las técnicas de análisis estructural y han colaborado en el refinamiento de los procedimientos de diseño de construcciones resistentes a los efectos de los sismos. A pesar de muchos terremotos que han afectado ciudades en los últimos 50-60 años, muchos de los cuales han sido prolíficamente documentados, hay grandes vacíos y ciertos tipos de construcciones seriamente afectados u otras no afectadas, no alcanzan a conformar una muestra lo suficientemente documentada para demostrar de manera contundente si los procedimientos de diseños han sido exitosos o requieren modificaciones.

El día 16 de abril de 2016, a las 18H58, se produjo en el Ecuador un sismo de 7,8 grados en la escala de Richter, cuyo epicentro se localizó entre las provincias de Esmeraldas y Manabí, generando importantes pérdidas humanas, así como daños severos en la infraestructura pública y privada de las provincias de Esmeraldas, Manabí, Santa Elena, Santo Domingo de los Tsáchilas, la Ingeniería Forense 
como herramienta de investigación nos permitirá ver los errores cometidos en la ejecución o en los diseños de la infraestructuras colapsadas en este evento telúrico.

\section{Clasificación de errores.}

Fallas de seguridad son los que provocan muerte, heridas o ponen a las personas en riesgo de ello incluyen el fallo catastrófico como, por ejemplo:

- Deficiencias del núcleo resistente

- Columna corta

- Columna débil y viga fuerte

- Discontinuidad estructural

- Golpeteo entre estructuras

- Piso débil

- Punzonamiento

- Muros de fachada de mampostería

- Cimentaciones

- Configuraciones estructurales en planta y en altura

- Tuberías embebidas en elementos estructurales

- Puentes.

\subsection{Deficiencias del núcleo resistente}

En los edificios de hormigón armado, el material de construcción debe cumplir varios requerimientos para que las estructuras resistan un sismo fuerte. El hormigón reforzado es un material mixto: está compuesta por hormigón y acero de refuerzo. El primer requerimiento refiere al hormigón, que resulta de la mezcla apropiada de cemento hidráulico, agregado grueso y agregado fino en condiciones controladas; para que el conjunto sea de calidad, ambos elementos constituyentes deben tener control en obra y cumplirse. Durante mucho tiempo los hormigones fabricados en obra han generado problemas de calidad como, por ejemplo: mala compactación, mal diseño de hormigones, segregación, poco material cementante, alta relación agua/cemento. entre otros factores que han ocasionado la mala calidad de hormigón fabricado en obra.

Adicionalmente, el mal vibrado del hormigón cuando se funden las secciones en obra por falta de control, y las malas condiciones de colocación generan secciones de hormigón con oquedades, hacen reducir el área neta de las columnas o vigas, que generan problemas frecuentes al reducir tanto la rigidez como la resistencia; esto es un fuerte problema actual debido a la falta de control en obra.
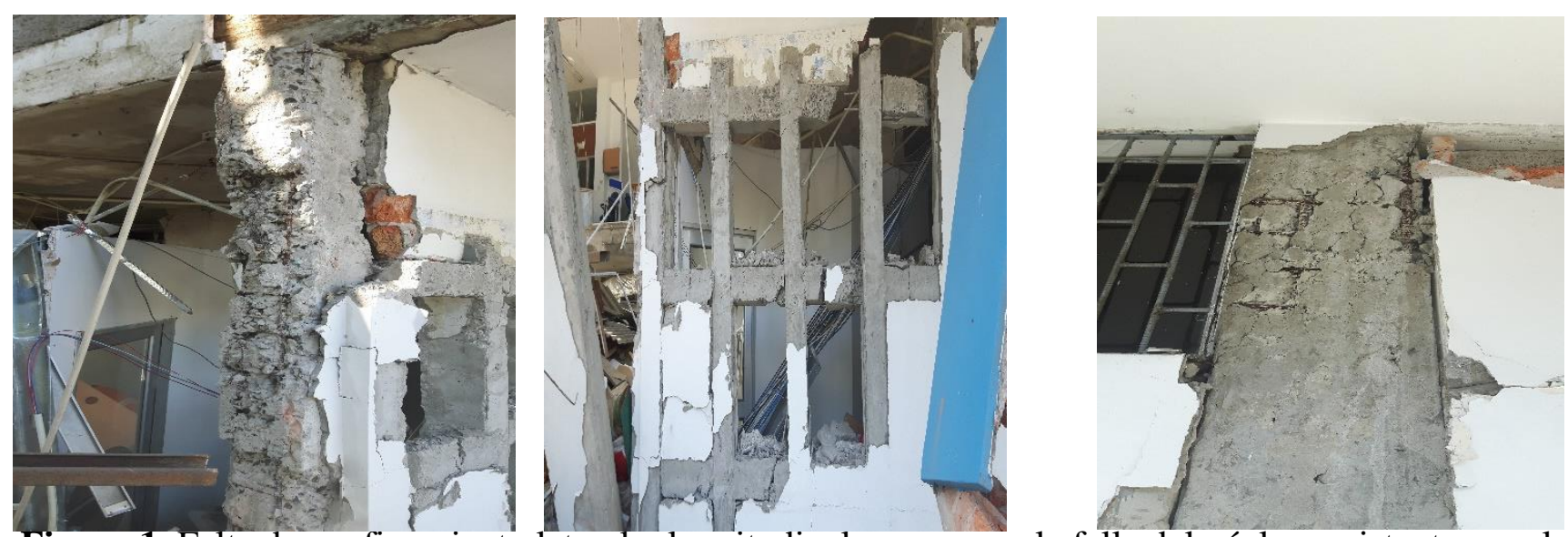

Figura 1. Falta de confinamiento lateral y longitudinal que genera la falla del núcleo resistente y mal vibrado de los elementos estructurales. 


\subsection{Columna corta}

Otro componente de vulnerabilidad estructural que se ha observado en el sismo del 16 de abril del 2016 y está muy documentado a partir de fallas reales y muchos ensayos de laboratorio se refiere a las denominadas columnas cortas, muy frecuente en construcciones hospitalarias o escolares. En general, la columna corta es una columna que hace parte del sistema estructural inicialmente libre de muros divisorios. Una vez terminada la construcción, los muros divisorios llegan hasta una cierta altura de la columna restringiendo su posibilidad de rotación. Si los muros son relativamente fuertes, la altura real de la columna frente a las fuerzas inerciales horizontales no es la del análisis, si no la libre por sobre la restricción dada por el confinamiento de los muros. El cortante absorbido por la columna resulta mucho mayor que el previsto, al tiempo que su capacidad de rotación se ve drásticamente disminuida. La combinación de estos factores produce fallas explosivas de la parte libre de la columna que se han observado en muchas partes del mundo.

El Ingeniero estructural no toma en cuenta los muros para su análisis y si algún día los pudiera tomar en cuenta, de poco le serviría porque en cuanto a muros y fachadas de ladrillo lo que hoy está en una edificación mañana puede ser cambiado por un propietario que decide hacer remodelaciones sin permiso de nadie. Separar los muros de la columna mediante la adición de una lámina de plástico esponjoso es una manera de resolver esta patología sísmica.
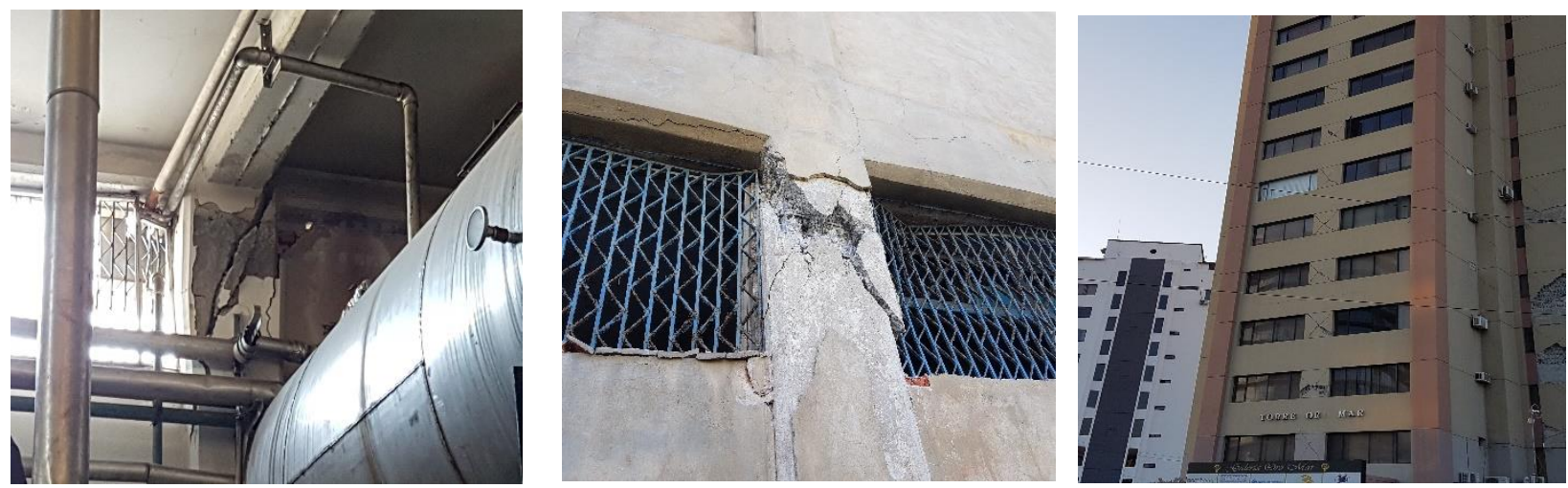

Figura 2. Edificaciones de recién construcción, hechas con técnicas sismorresistentes modernas, sufren de las consecuencias de la columna corta por presencia de ladrillo hasta cierta altura.

\subsection{Columna débil y viga fuerte}

Esta patología sísmica es muy peligrosa porque la columna da soporte a las vigas que convergen al nudo. Desde este punto de vista, la falla de una columna es intrínsecamente más peligrosa que la falla de una viga. Al producirse las fuerzas inerciales que generan los desplazamientos horizontales de las construcciones, los nudos sufren rotaciones cuyos efectos se reparten a los elementos estructurales convergentes en proporción a su rigidez. Si las vigas son muchos más rígidas que las columnas, para la columna la situación es equivalente a aquellas en la cual estuviera empotrada en la viga y, en consecuencia, la rotación resultante sobre ella es más marcada que la que ocurriría si la viga hubiera podido rotar un poco. En estos casos se precipita la falla de la columna afectando además de las vigas que convergen al nudo perdiendo su sustentación. 

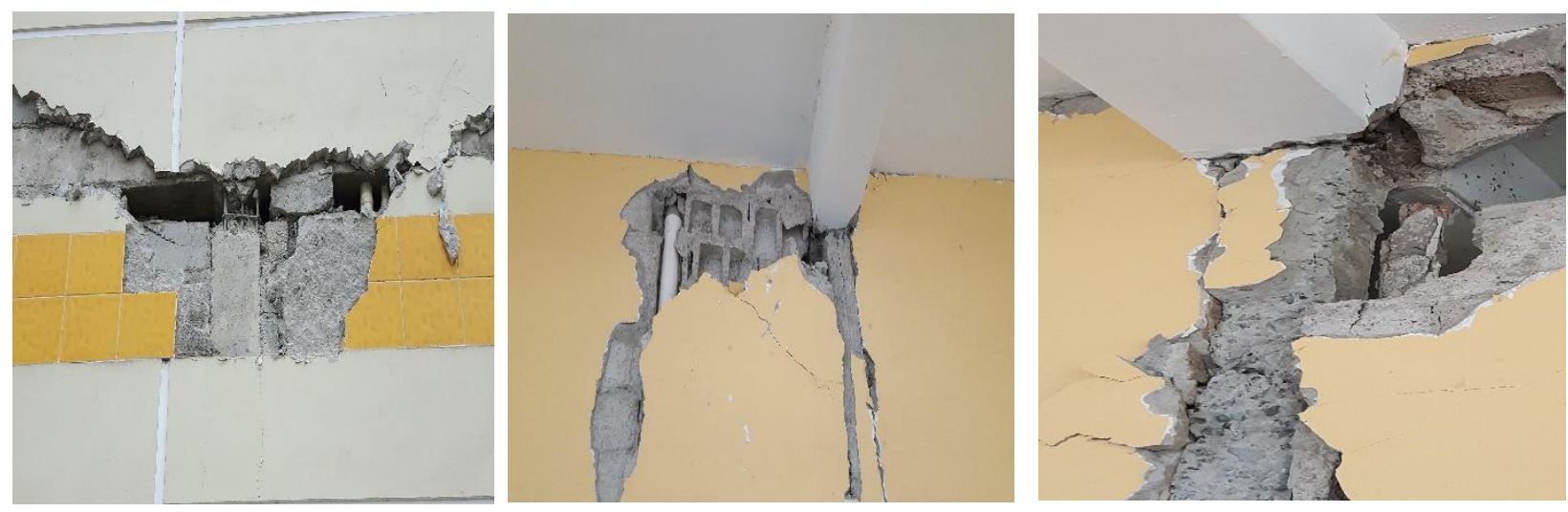

Figura 3. Columna débil bajo una viga fuerte, se evita este daño diseñando columnas con suficiente rigidez y resistencia a partir de secciones más generosas en cuanto a hormigón y acero de refuerzo longitudinal y transversal.

Lo que se busca es la articulación, pero de la viga, la cual deberá tener mucha menor rigidez rotacional y resistencia que la columna conectante. Si no se cumple, el daño se va hacia la columna y poco se puede hacer. Esto se presentaba comúnmente en columnas construidas para edificaciones hechas para soportar solo carga vertical.

\subsection{Discontinuidad estructural}

La presencia de pisos intermedios y también de escaleras con descansos que se apoyan en columnas o la mala práctica de ubicar masas especiales como puede ser piscinas o depósitos en pisos intermedios de las edificaciones suelen conducir a discontinuidades estructurales. Otra práctica constructiva que genera discontinuidades corresponde a cambios significativos de la rigidez de las columnas al pasar de un determinado piso al siguiente. Durante la construcción se puede adicionar rígidos y frágiles muros de ladrillo que generan una discontinuidad estructural que concentre la demanda ductilidad sobre la columna.

También ocurren discontinuidades estructurales cuando las vigas son excéntricas con respecto a la columna, por ejemplo, vigas que se apoyan sobre una parte de la columna e inclusive casos en las cuales la viga prácticamente es un apéndice de la columna.
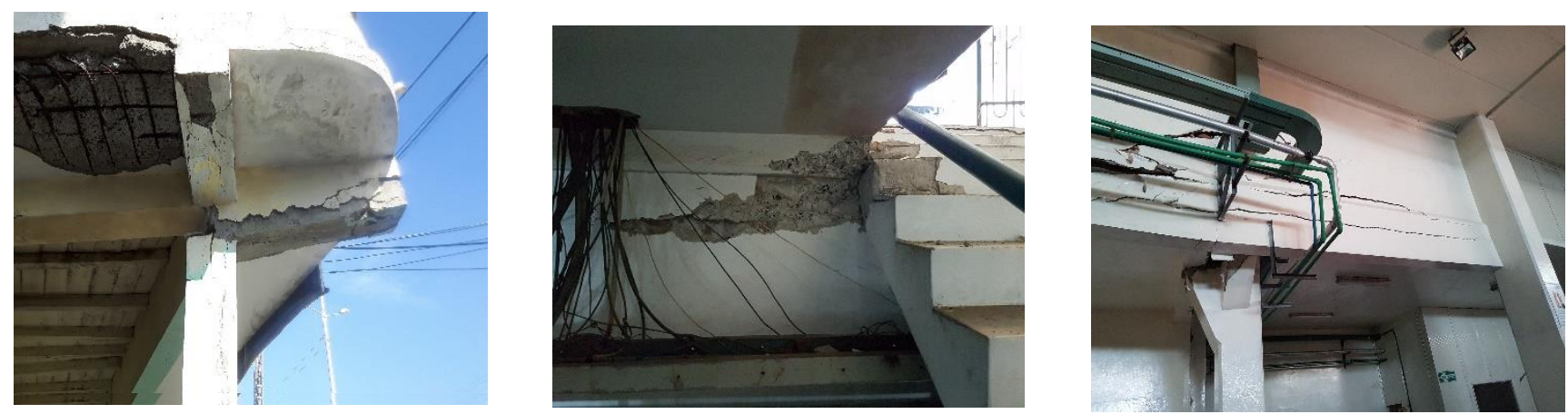

Figura 4. Cambios bruscos de secciones de vigas en nudos, falta de continuidad del acero de refuerzo, creación de cartelas en medio de columna.

Mantener la continuidad en cuanto a secciones de hormigón y acero a lo largo de la altura de toda la columna parece ser todo el secreto para evitar esta patología sísmica. 


\subsection{Golpeteo entre estructuras}

En muchas ocasiones, edificios de diferente altura, masa y rigidez son construidas unos junto a otros de tal manera que cuando son sometidos a movimientos sísmicos los patrones de desplazamiento de cada estructura son diferentes; esto ocasiona

Que en algún momento de la historia del sismo, los edificios se golpeen fuertemente destruyéndose mutuamente, sobre todo si las placas de entrepiso no se encuentran a la misma elevación; este fenómeno denominado golpeteo se ha presentado en sitios con alta densidad poblacional donde construyen los edificios sin ningún tipo de separación.
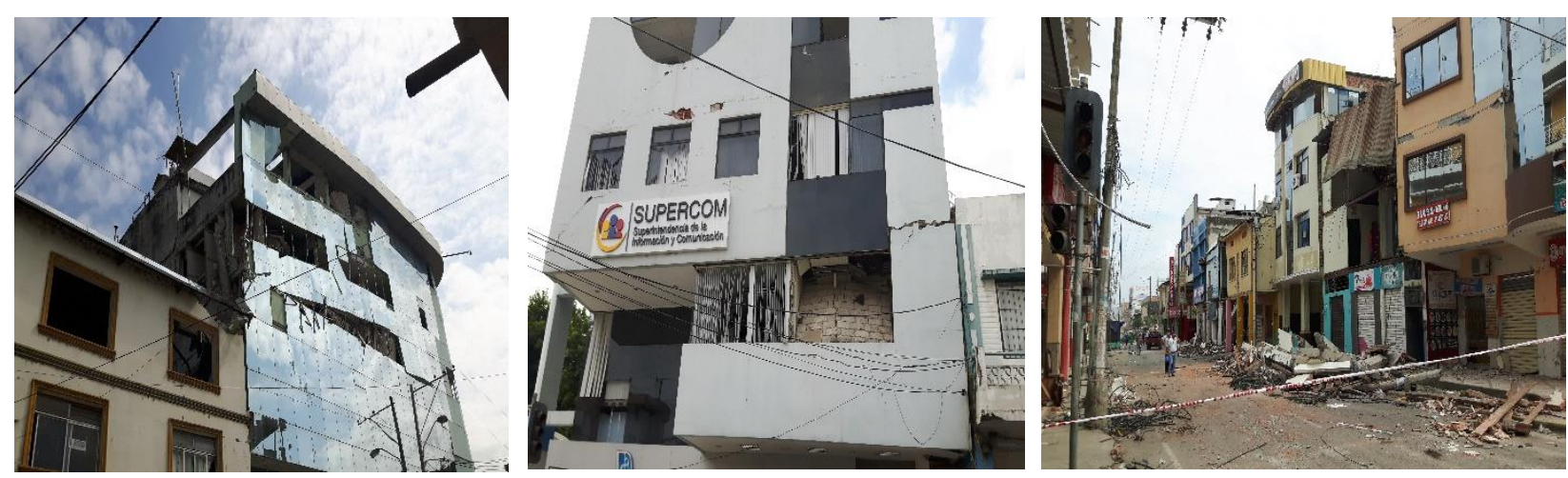

Figura 5. El problema sigue creciendo día a día, porque edificaciones nuevas se siguen construyendo pegadas a las antiguas.

\subsection{Piso débil}

Existe preocupación por este fenómeno generalizado, que logra que una edificación bien construida, con buenos materiales sufra daño irreparable en sus primeras plantas, y por ende, pérdida total.

Cuando una edificación tiene muros divisorios de ladrillo a partir del primer nivel y no los tiene en la planta baja, en la primera losa se concentra una muy elevada demanda de ductilidad al oscilar la estructura durante un sismo intenso. Esta discontinuidad deriva de la alta rigidez de la edificación desde la primera losa hacia arriba comparada con la rigidez en la planta baja, implica que la parte con muros del edificio tienda a oscilar como una caja muy rígida construida sobre un sistema mucho más débil que es lo correspondiente a la planta baja. La consecuencia de esta situación es que las columnas no pueden suministrar las rotaciones necesarias y fallan por una demanda de ductilidad por fuera de sus posibilidades de capacidad dúctil; es una falla frágil y rápida, no tiene posibilidad de reparación alguna.
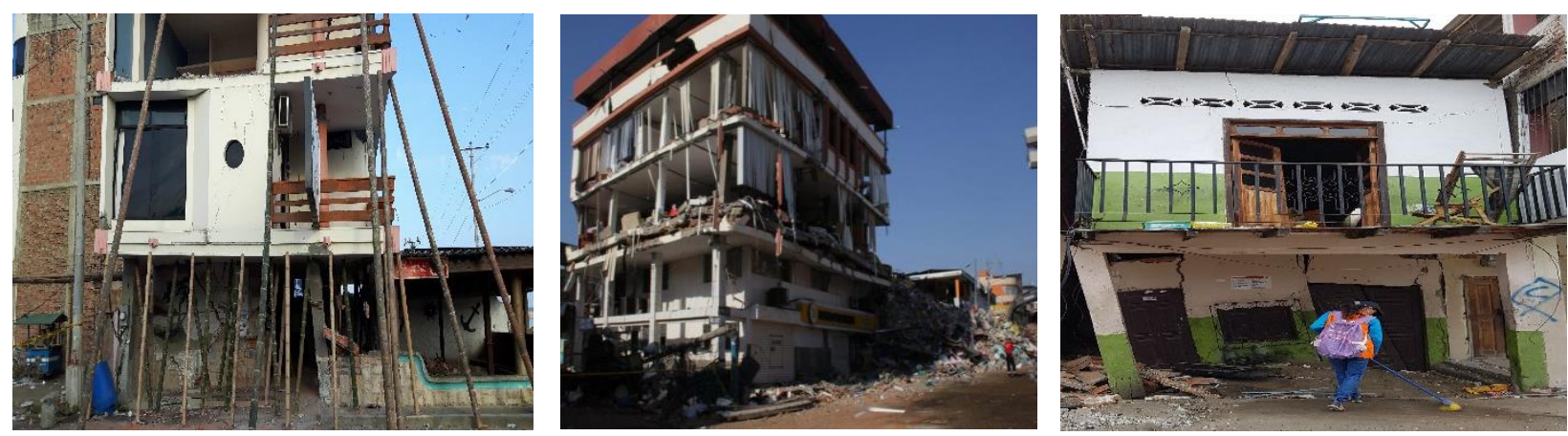

Figura 6. Colapso del primer piso por configuración de un piso débil, también hay un piso débil en un entrepiso superior. Los pisos bajos con pocas paredes se deben a circunstancias administrativas o simplemente para ubicar parqueaderos y áreas sociales abiertas. 


\subsection{Punzonamiento}

En las estructuras de losa plana y aligeradas en dos direcciones, y en cualquier losa, se desarrolla una fuerte cortante perimetral alrededor de la columna correspondiente a la acción gravitatoria. Cuando ocurre un sismo la solicitación dinámica proveniente de los desplazamientos horizontales de la edificación se suma con la solicitación estática y se incrementa notablemente el efecto de cortante sobre la losa en las inmediaciones de la columna.
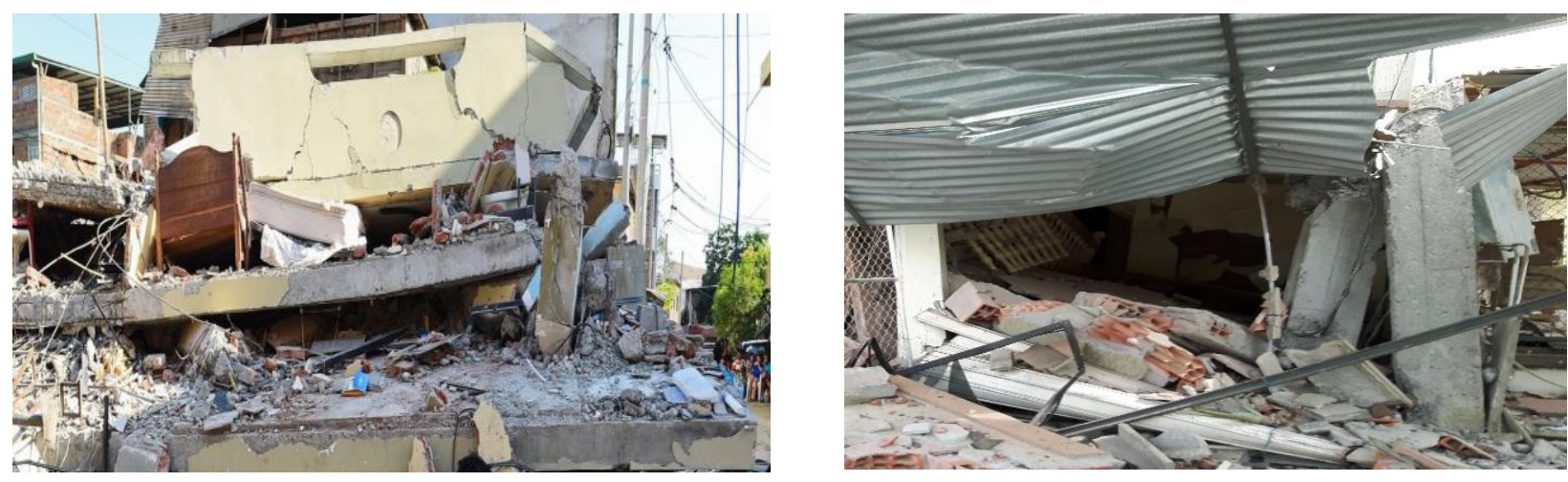

Figura 7. Punzonamiento de losas planas de dos direcciones, la perdida es total. Al ocurrir el sismo y desarrollarse los desplazamientos horizontales producidos por las fuerzas inerciales.

\subsection{Muros de fachada de mampostería}

Las patologías de agrietamiento de los muros y fachadas de ladrillo o bloque es posiblemente la más común en edificaciones estructuradas durante la ocurrencia de un sismo. Los pórticos con muros de relleno se vuelven más rígidos una vez que la estructura hace contacto con el tabique de relleno, incrementando la rigidez y cambiando las propiedades dinámicas de la estructura. Cuando las mamposterías están distribuidas en forma asimétrica, pueden ocasionar vibraciones torsionales, dando lugar a incrementos de los esfuerzos cortantes sobre la estructura. Estas mamposterías se agrietan severamente si no han sido diseñados para soportar estas fuerzas e incluso pueden causar un efecto muy nocivo, pues dichas fuerzas pueden también producir graves daños en la estructura misma.
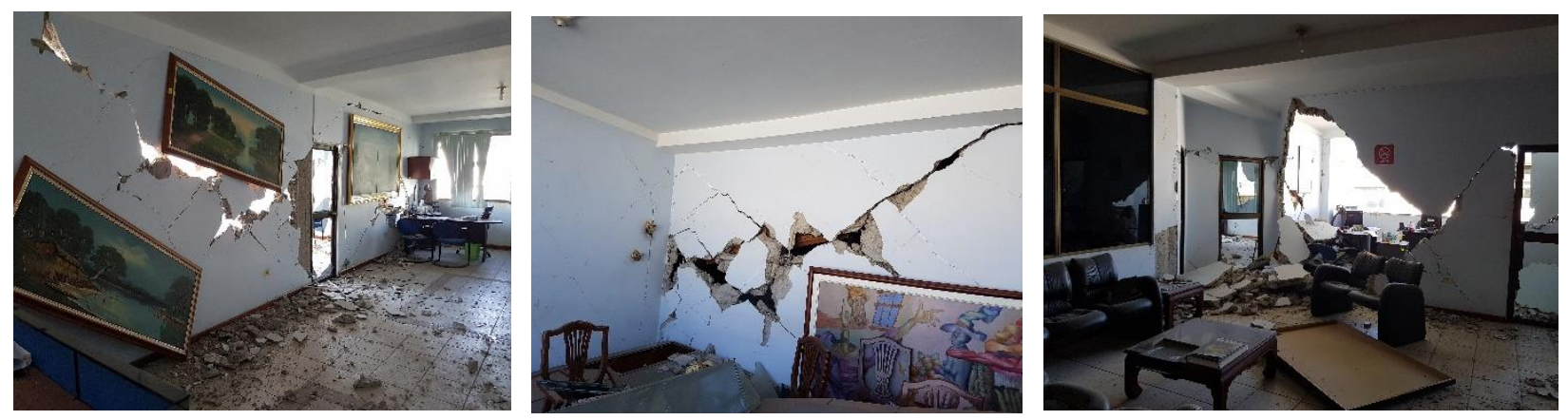

Figura 8. Desprendimiento de mamposterías por falta de confinamiento.

\subsection{Cimentaciones}

Cuando el análisis y el diseño de la cimentación se han concentrado primordialmente en la situación estática y se ha forzado la situación hasta producir cimentaciones en las cuales el esfuerzo de contacto o la carga sobre la cimentación resulta anormalmente elevado, el comportamiento estático de la edificación puede resultar satisfactorio. Sin embargo, cuando actúan las fuerzas inerciales producidas por un sismo, la situación estática forzada que ya existía se manifiesta en asentamientos diferenciales sobre la construcción que la dejan con una deformación residual en su cimentación mostrando una 
inclinación del edificio tan marcada que es alarmante. Entre otras situaciones más graves, el edificio simplemente se vuelca. La susceptibilidad a la licuación de un suelo depende de las condiciones que se han anotado, pero, además, de las características del sacudimiento sísmico. En cuanto al sismo, se ha establecido que la aceleración local y su permanencia entendida como la posibilidad de que ocurra un mínimo número de ciclos de alta aceleración, son condiciones necesarias para que se desarrolle la licuación, induciendo la patología de asentamiento o volcamiento de las edificaciones.

Obras portuarias que incluyen muchas construcciones de hormigón reforzado o vías importantes, se han visto gravemente afectadas por la patología de la licuación de suelo.
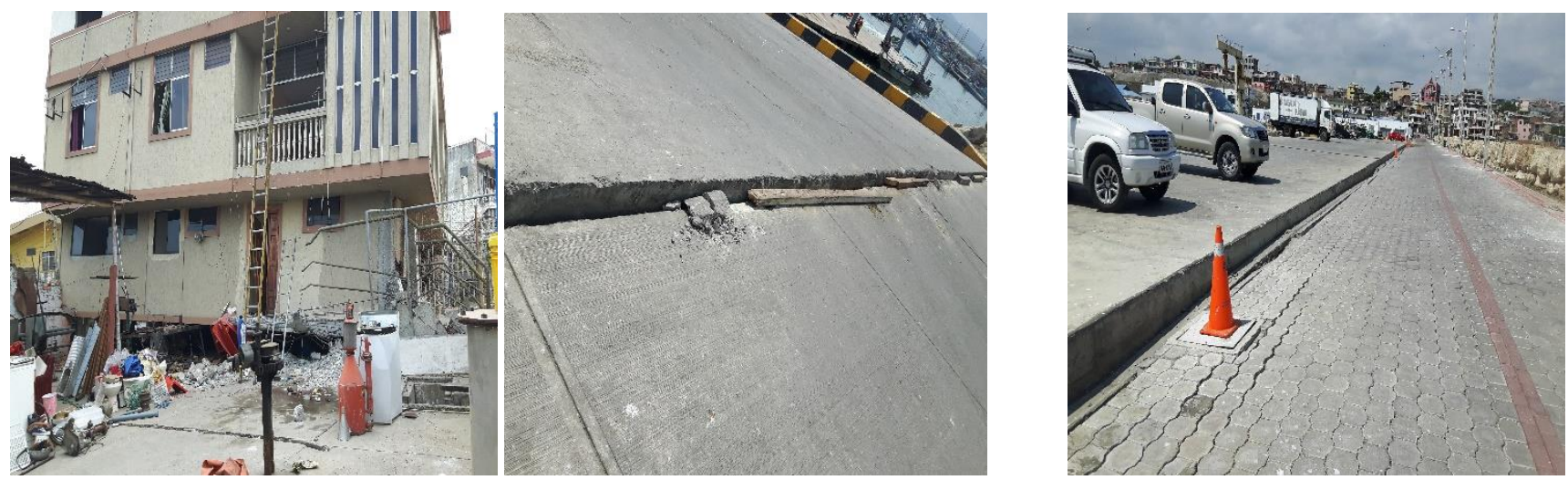

Figura 9. Colapso de la edificación por falla de la capacidad portante de la cimentación, vías, obras portuarias con pérdida de capacidad portante debido a la licuación.

\subsection{Configuraciones estructurales en planta y en altura}

Las irregularidades estructurales en planta y en altura han sido la causa de patologías que producen daños graves. Las irregularidades inducen comportamientos dinámicos complicados en las cuales la edificación genera fuerzas inerciales que pueden producir deformaciones excesivas difíciles de prever con daños en muros, torsión en planta y en otros casos han conducido al colapso de la edificación. Como el momento de torsión mencionado genera fuerzas sísmicas adicionales a las inerciales en cada dirección principal de la edificación, el diseñador debe asegurar no solo la rigidez necesaria para controlar las deformaciones transversales de la estructura, sino que el centro de rigidez conduzca a una excentricidad mínima, por consiguiente, tanto el centro de masa como el de rigidez son variables.
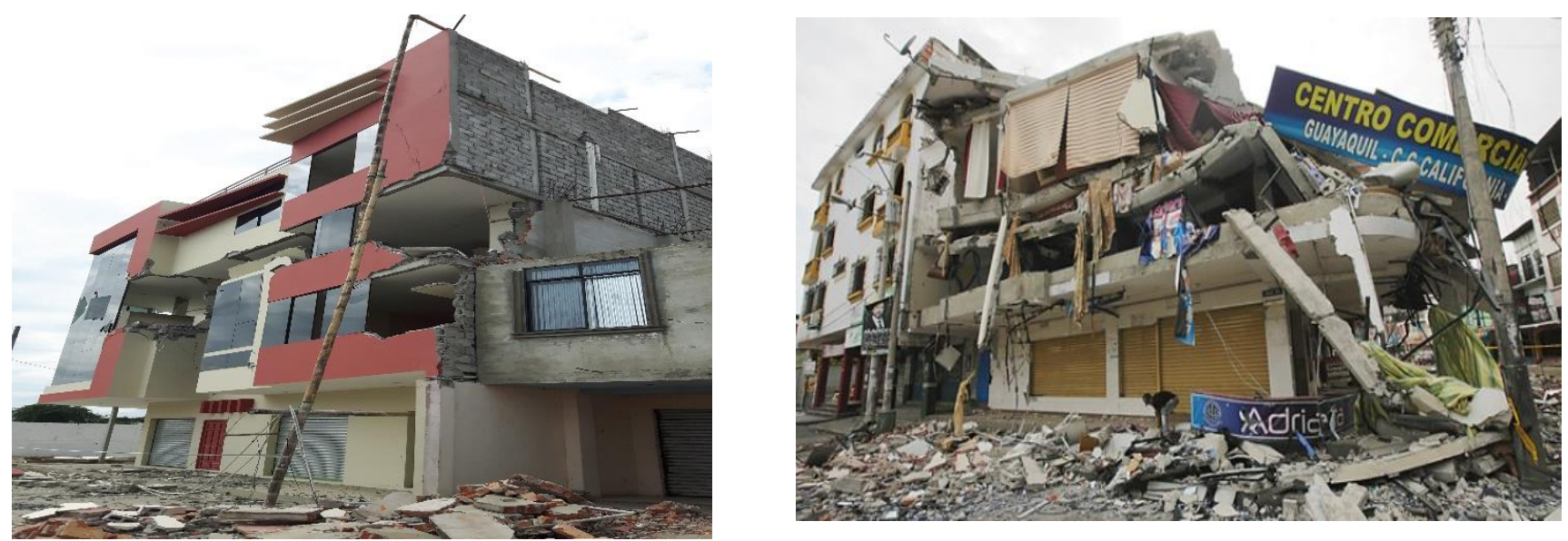

Figura 10. Edificaciones con irregularidades en planta que tuvo fuertes problemas por la torsión generada. 


\subsection{Tuberías embebidas en elementos estructurales}

El hecho de hacer perforaciones en los elementos estructurales de soporte es una práctica que atenta contra los sanos principios de estructuración, ya que por medio de estas prácticas los elementos pierden drásticamente su sección y su inercia y por ende la rigidez, resistencia y capacidad dúctil. Hacer una perforación en un elemento o embeber una tubería, equivale a pre fallar la sección, la cual será inútil cuando realmente se necesite de ella en un sismo. Si la práctica se repite a lo largo de toda la edificación, las posibilidades para soportar un fuerte evento telúrico, se ven reducido.

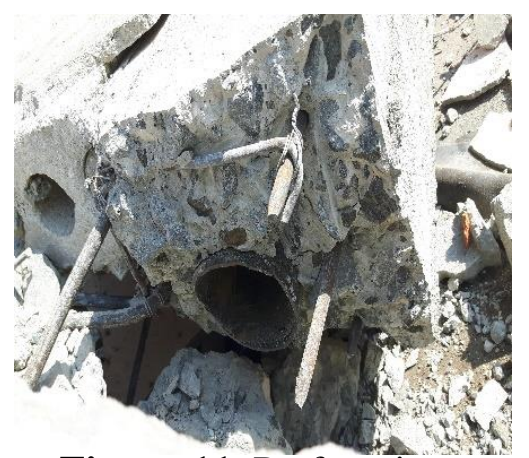

Figura 11. Perforaciones en columnas y tuberías embebidas,

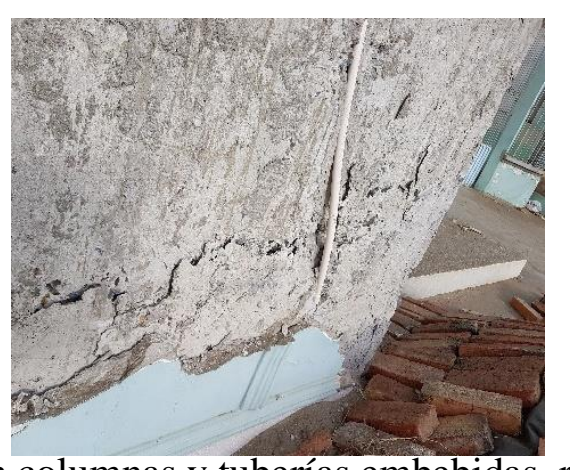
patologías congénitas que hicieron fallar a la estructura en el sismo del 16 de abril del 2016.

\subsection{Puentes}

Un puente es una obra esencialmente lineal, puede atravesar una zona de falla de tal manera que al producirse la ruptura, las columnas del puente no solo sufren el sacudimiento producido por el paso de las ondas sísmicas sino que si la fractura llega a la superficie del terreno, puede suceder que una columna se desplace con respecto a la otra. Dependiendo de la geometría del puente, se puede generar una patología desastrosa para la obra que la lleve a la destrucción total.
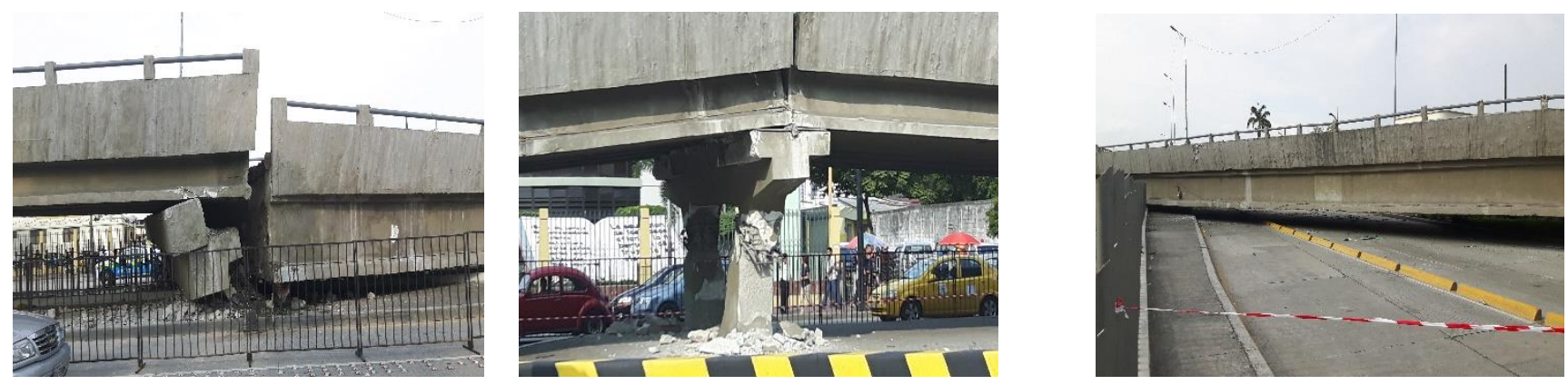

Figura 12. Colapso del puente por fallas en las columnas por cambio brusco de sección, falta de refuerzo longitudinal y transversal, así como también la baja resistencia del hormigón.

\section{CUALIDADES DEL INGENIERO FORENSE.}

Ser objetivos en la investigación como es:

a. Buena formación académica.

b. Experiencia profesional comprobada.

c. Resolución de conflictos en casos similares.

Poseer ética profesional en la investigación como es:

a. Veracidad en la información.

b. Objetivo en los resultados.

c. Ser imparcial en las conclusiones finales 


\subsection{Investigación Forense}

Son varios los métodos usados para una investigación entre los cuales tenemos:

Método racional.

$>$ Prioridades (tareas).

$>$ Equipos especializados.

$>$ Investigación ocular (inspecciones).

$>$ Testimonios (testigos oculares y muestras).

$>$ Documentación.

$>$ Hipótesis.

$>$ Ensayos varios.

$>$ Análisis de los resultados.

$>$ Conclusiones.

Las actividades del Ingeniero Forense comprenden lo siguiente aspectos:

a. Observación del escenario del colapso de la estructura.

b. Identificar las pistas.

c. Croquis del colapso (fotos planos,)

d. Protección de evidencias.

e. Numeración de fotografías.

f. Establecer el siniestro con sistemas de coordenadas georeferenciales.

Dentro de los testimonios se debe entrevistar inmediatamente y por separados entre los actores principales tenemos:

a. Obreros de la construcción objeto de investigación.

b. Transeúntes que observaron el siniestro y vecinos que miraron el evento.

c. Recabar fotografías de los testigos que pudieron tomar fotos en el instante del siniestro.

\subsection{Recolección de evidencias}

Las evidencias deben ser marcadas, identificadas, catalogadas y almacenada en lugar muy seguro y organizado. Y debe ser revisada por el Ingeniero Forense, se debe tratar las evidencias con mucho cuidado, hay que evitar el manipuleo, empaquetar con materiales que no comprometan las evidencias, el transporte de evidencias debe tomar un tiempo establecido para llegar al laboratorio si no las muestras no tendrán valides.

Cuando se trata de recolección de documentos tenemos que recopilar los siguientes:

a. Documentos de diseño entre los cuales son: cálculos estructurales memorias de cálculo, normas y códigos utilizados en el diseño.

b. Planos y especificaciones de construcción.

c. Planos de construcción (detalles constructivos).

d. Contratos de trabajo.

e. Informes de laboratorio entre los cuales tenemos: cimentación, resistencia del Hormigón, ensayos de acero, entre otros ensayos.

f. Certificados de calidad entre los principales son: cemento laboratorios hierro.

g. Informes de fiscalizador.

h. Seguridad laboral certificaciones, afiliaciones de seguros entre otros.

\subsection{Aspectos legales.}

Cuando ya ingresamos en aspectos legales y/o disputas tenemos los siguientes:

a. Litigación penal o civil tenemos: perito de parte, perito judicial.

b. Resoluciones alternativas tenemos: peritaje, negociación. 
En todos los casos la colaboración del Ingeniero Forense es muy valiosa. En los aspectos legales hay que ser concisos, no contradecir con los Informes y documentación usada para los Informes previos, hablar claro, esperar que las preguntas estén bien formuladas.

\subsection{Revisión de antiguas penas}

En realidad, muchos de los actuales criterios de diseño para garantizar seguridad estructural se basan en siglos de costumbres; desde tiempos atrás la sociedad ha colocado especial énfasis en garantizar de alguna forma la integridad de sus ciudadanos, y lo ha hecho a través de regulaciones. En un principio los mínimos requisitos de seguridad fueron especificados por severas penalidades para los constructores que no construían en forma segura. El más antiguo código de construcción del que se tiene noticia fue usado en Mesopotamia e instaurado por Hammurabi, Rey de babilonia, quien murió hacia 1750 antes de Cristo. Las reglamentaciones de este código fueron labradas en una roca que hoy es preservada en el museo de Louvre en París, Francia, en dichas rocas dice lo siguiente:

LEY 229: Si un constructor construye una edificación para alguien y su estructura no es lo suficientemente fuerte y la casa se derrumba causando la muerte del dueño, el constructor debe ser ejecutado.

Ley 230: Si el hijo del propietario murió, se matará al hijo del constructor.

Ley 231: Si murió el esclavo, el constructor dará otro esclavo equivalente al propietario.

Ley 232: Si se perdió todos los bienes por el colapso, el constructor pagará todo y construirá otra casa a su propia cuenta.

\section{CONCLUSIONES}

El objetivo de la construcción es mejorar las condiciones de vida de la gente llenando sus necesidades básicas. El resultado hará posibles nuestras diarias tareas materiales y espirituales. El constructor es un servidor de la sociedad, desarrollador de la cultura y de la calidad de vida. Nótese que en el código de Hammurabi, la responsabilidad recaía directamente sobre el constructor y la capacidad de creación y el ingenio eran libres. Nótese que el código de Hammurabi no se le enseñaba al constructor a construir y mucho menos lo obligaba a usar alguna técnica particular de construcción. En los códigos actuales ocurre exactamente todo lo contrario, el ingenio y la creación son limitados a un articulado, que le indica al profesional como debe proyectar y lo obliga hasta usar ecuaciones prescritas

\section{BIBLIOGRAFÍA}

Gallego, M., Sarria, A., (2006), "El concreto y los terremotos" (Instituto del Concreto, Asocreto), p. $185,248$.

Morocho, T., (2016). “Archivo fotográfico recopilado después del sismo del 16 de abril de 2016 ocurrido en Ecuador", 2016.

Morocho, T., "Patología y durabilidad del hormigón estudios casos", Tesis Doctoral, Atlantic International University, (2017) p. 126, 133. 